\title{
Decomposing and Sketching 3D Objects by Curve Skeleton Processing
}

\author{
Luca Serino, Carlo Arcelli, and Gabriella Sanniti di Baja \\ Institute of Cybernetics "E. Caianiello", CNR, Naples, Italy \\ \{1.serino, c.arcelli,g.sannitidibaja\}@cib.na.cnr.it
}

\begin{abstract}
A 3D object decomposition method is presented, based on the polygonal approximation of the distance labeled curve skeleton. Polygonal approximation is accomplished to divide each skeleton branch into a number of segments along which no significant changes exist as regards curvature or distance label. Each segment is interpreted as the spine of a simple region, which is characterized by i) absence of significant curvature changes along its boundary and ii) thickness that is either constant or evolves linearly along the region. Quantitative information on shape, size, position and orientation of a simple region can be easily derived from spatial coordinates and distance labels of the extremes of the associated spine. Simple regions associated to spines sharing a common extreme partially overlap with each other. Object decomposition into disjoint regions is obtained by suitably dividing each overlapping region among the simple regions including it.
\end{abstract}

Keywords: Object decomposition, curve skeleton, distance information, polygonal approximation.

\section{Introduction}

Decomposition is of interest for 3D object recognition, especially when the structural approach is followed. A 3D object having complex shape can be first decomposed into parts characterized by simpler shape. Then, the description of the object, leading to its recognition, can be given in terms of the descriptions of the obtained parts and of the spatial relationships among them.

Different approaches to object decomposition have been suggested in the literature. For example, decomposition can be achieved by identifying in the object a priori defined shape primitives, such as balls, cylinders, cones, prisms and so on. Alternatively, if the object is represented by its boundary surface, the parts can be achieved by using cutting planes passing through curvature minima suitably identified along the boundary surface. Finally, if the object is represented by its skeleton object decomposition can be obtained by decomposing the skeleton in such a way that each part of the decomposed skeleton corresponds to a part of the object.

The skeleton of a 3D digital object consists of the voxels that are placed symmetrically within the object. If objects have tubular shape, symmetry points are mainly aligned along symmetry axes, so that the skeleton is a set of curves. 
For objects with general shape, symmetry points are placed along axes and planes, so that the skeleton is union of surfaces and curves. In this work, we consider objects that can be reasonably represented by curve skeletons, i.e., skeletons exclusively consisting of curves.

Each branch of the skeleton is in correspondence with a part of the object. If the skeleton branch includes an end point, i.e., a voxel having only one neighbor in the skeleton, the branch is a peripheral branch and corresponds to a limb of the object. Otherwise, the branch is an internal branch and is in correspondence with a part of the object, here called core, from which limbs protrude.

The curve skeleton is homotopic to the object and, if its voxels are labeled with their distance from the complement of the object, the object can be recovered by the envelope of the balls centered on the skeleton voxels and having radii equal to the distance values assigned to the skeleton voxels. Actually, a difference exists between an input object and the recovered object. Such a difference is negligible only when the skeleton includes almost all the symmetry points. In all other cases, only a sketched version of the input object can be recovered by the skeleton. As an example, Fig. 1 shows from left to right an object, its curve skeleton and the object recovered by the skeleton. In this case, about $74 \%$ of input object voxels are recovered by the skeleton. Thus, the recovered object, which is faithfully represented by the curve skeleton, is a reasonable sketched version of the input object.
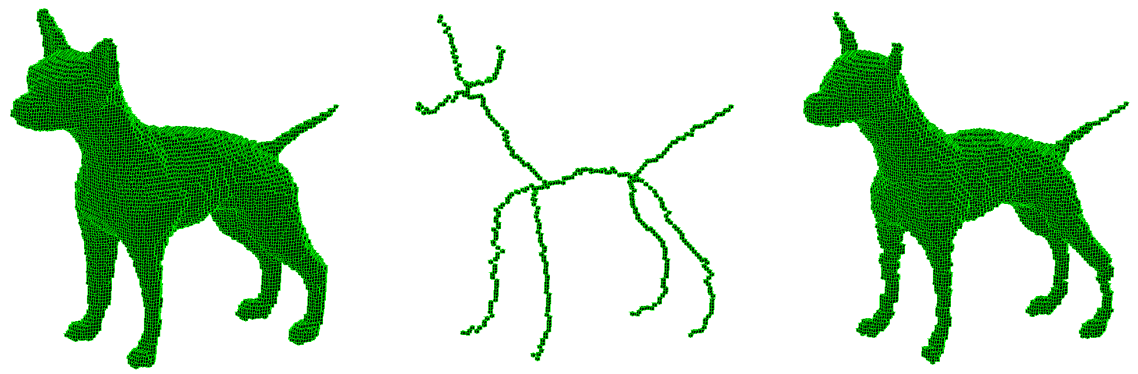

Fig. 1. An object, left, its curve skeleton, middle, and the recovered object, right

The curve skeleton can provide a representation of the input object at different levels of detail by using pruning or polygonal approximation. Pruning can be used to trim skeleton branches corresponding to limbs interpreted as object regions having scarce perceptual relevance. If a suitable parameter is introduced to measure perceptual relevance of object regions via the analysis of the skeleton branches mapped into those regions, skeleton pruning can originate different results by using different thresholds for the parameter. In this way, different recovered objects can be obtained, which are sketches at different levels of detail of the input object. In particular, recovered objects differ from each other for the presence/absence of some peripheral regions. Alternatively, each branch of the skeleton can be divided into a number of segments by means of polygonal approximation. In this case, each segment can be seen as the spine of a region of the object, whose boundary is rid of significant curvature changes in the limits of the adopted tolerance. By using different thresholds 
for the polygonal approximation, different representations of the object are obtained: no object's limbs are lost, while the geometry of each object part is represented in a more or less faithful manner.

In this paper, we continue our work concerning 3D object decomposition via skeleton processing [1]. Here, we decompose 3D objects by decomposing their curve skeletons. We use the 3D skeletonization algorithm described in [2], which is based on the extraction of the curve skeleton from the $<3,4,5>$-distance transform of the object. We divide the skeleton into its constituting branches, identify only the branches corresponding to meaningful object parts, and perform polygonal approximation on such branches so as to divide them into segments. The regions whose spines are the so obtained segments are sketched versions of the regions into which the object can be interpreted as decomposed.

\section{Preliminaries}

Let us consider a binary voxel image in a cubic grid, where the object $A$ consists of the voxels with value 1 , while the background $B$ consists of the voxels with value 0 . The $3 \times 3 \times 3$ neighborhood of a voxel $p$ includes the six face- the twelve edge- and the eight vertex-neighbors of $p$.

The $<3,4,5>$-distance between two voxels $p$ and $q$ is given by the length of a minimal path from $p$ to $q$, where the three weights 3,4 and 5 are respectively used to measure the unit moves from $p$ towards a face-, edge- and vertex-neighbor along the path [3].

The $<3,4,5>$-distance transform of $A$ is a replica of $A$, where the voxels are labeled with their $<3,4,5>$-distance from $B$.

The curve skeleton $S$ of $A$ is homotopic to $A$, consists of curves centered in $A$, and its voxels are labeled with their distance from $B$. A voxel $p$ of $S$ having in $S$ only one neighbor, exactly two neighbors, and more than two neighbors is respectively termed end point, normal point, and branch point.

Any connected subset of $S$ entirely consisting of normal points, except for the two extremes that are end points or branch points is termed skeleton branch. The skeleton branch is an internal branch if both extremes are branch points, and is a peripheral branch otherwise.

The reverse distance transform of $S$ is the envelope of the balls centered on the voxels of $S$ and having radii equal to the corresponding distance values [4]. We use the $<3,4,5>$-distance also to compute the reverse distance transform of $S$.
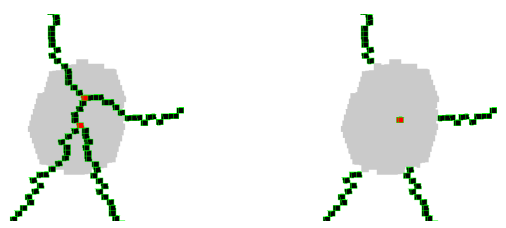

Fig. 2. Left, a skeleton (green) with a zone of influence (gray) including two branch points (red). Right, the centroid (red), common to the four meaningful branches (green). 
Let us consider the balls associated with the branch points of $S$. Each connected component of balls is called zone of influence of the branch points it includes. Branch points that are neighbors of each other or are closer to each other than the sum of their associated radii are included in the same zone of influence. Actually, these close branch points of $S$ can be understood as corresponding to a unique branch point configuration in an ideal skeleton of the object, and the short skeleton branches linking close branch points can be interpreted as non perceptually meaningful. Accordingly, in the following we replace all the voxels of $S$ that are included in the same zone of influence by their centroid. The centroid plays the role of an ideal branch point, where skeleton branches of the ideal skeleton meet. We regard as meaningful skeleton branches of $S$ the sets consisting only of the skeleton voxels outside the zones of influence plus the corresponding centroids. See Fig. 2.

We use the algorithm [5] to compute the polygonal approximation of skeleton branches. The process is as follows. The extremes of the current digital curve are taken as vertices; the Euclidean distance of all points of the curve from the straight line joining the two extremes is computed; the point with the largest distance is taken as a new vertex, provided that such a distance overcomes an a priori fixed threshold $\theta$ (to be set depending on the desired approximation quality). Any detected vertex divides the curve into two subsets, to each of which the above process is applied. The splitting process is repeated as far as new vertices are detected. When recursion is completed, the curve is represented by the ordered sequence of the detected vertices.

\section{The Decomposition Method}

Generally speaking, $S$ includes a number of meaningful branches. For the sake of simplicity, let us describe our method by referring to a simple case where the skeleton consists of a single branch. See Fig. 3. We observe that curvature changes along $S$ correspond to bendings of the object. Then, polygonal approximation can divide $S$ into straight line segments, each of which can be seen as the spine of a region rid of bendings.
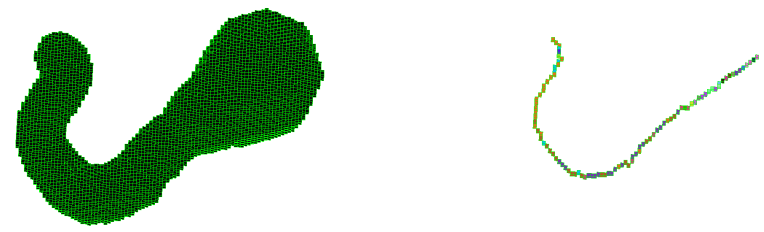

Fig. 3. An object, left, and its skeleton, right. Colors of skeleton voxels denote distance values.

We also note that the different radii of the balls associated with the skeleton voxels take into account the changes in width of the object. Let us consider the $2 \mathrm{D}$ representation of $S$ obtained by plotting $S$ in the Cartesian plane as a function of the radius associated to its voxels. If polygonal approximation is there performed, vertices are detected wherever the radii fail to be aligned in the limits of the adopted tolerance. Thus, the skeleton can be divided in such a way that the radii of the voxels in each 
segment are either constant, or change in a linear manner. Each segment can be interpreted as the spine of a region characterized by either constant or linearly increasing/decreasing thickness.

By considering at the same time changes along $S$ in geometry and in distance values, the polygonal approximation can divide $S$ into segments characterized by linearity both in geometry and in the distribution of distance values. Each segment of $S$ is the spine of a simple region characterized by the following two properties: 1) absence of significant bendings, and 2) thickness that either is constant or is linearly increasing/decreasing. In other words, a simple region is shaped as a cylinder or a cone, delimited by the two balls centered on the extremes of its spine. See Fig. 4.
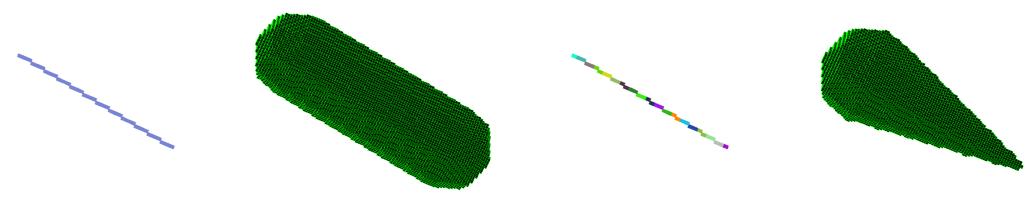

Fig. 4. Straight line skeleton segments and their corresponding simple regions. Colors of skeletal voxels denote distance values.

To reach the above goal, $S$ is represented in a 4D space, where the four coordinates are the three Cartesian coordinates and the radius of the voxels of $S$. To compute in such a space the polygonal approximation of $S$, we need to evaluate the Euclidean distance $d$ of any point $c$ of $S$ from the straight line joining the two extremes $v$ and $w$ of $S$. This is done by using the following expression:

$$
d^{2}=\|v c\|^{2}-\mathrm{P}_{v w c} * \mathrm{P}_{v w c} /\|v w\|^{2}
$$

where $\|v w\|$ is the norm of the vector $v w$, and $\mathrm{P}_{v w c}$ is the scalar product between vectors $v w$ and $v c$. If the point $c$ is the one at maximal distance from the straight line joining $v$ and $w, c$ is taken as a vertex of the polygonal approximation provided that its distance $d$ satisfies the condition $d>\theta$.

The vertices detected in the 4D space are used to identify in the $3 \mathrm{D}$ space the corresponding voxels of the skeleton. Accordingly, $S$ can be efficiently represented by orderly giving only the spatial coordinates and radii of these voxels.

When $S$ consists of more than one single branch, polygonal approximation has to be applied to all meaningful skeleton branches, so originating an approximated version of the entire skeleton.

An approximate evaluation of shape, size, position and orientation of the simple regions having the segments of $S$ as spines is readily available without the need of building the regions themselves. In fact, the shape of a simple region depends on whether the extremes of the spine are equally distance labeled (in this case the simple region is shaped as a cylinder, whose bases pass through the two extremes of the spine and are perpendicular to the spine) or have different values (in this case the simple region is shaped as a truncated cone, whose bases pass through the two extremes of the spine and are perpendicular to the spine). Of course, in both cases the shape of the simple region is completed by two semi-spheres placed on the bases of 
the cylinder or the truncated cone, where the radii are the distance labels of the extremes of the spine. Size can be measured by taking into account the length of the spine and the radii assigned to the extremes. Position and orientation are obviously related to the spatial coordinates of the extremes and to the orientation of the spine. The angle between two spines sharing a common extreme accounts for the bending of the two corresponding simple regions, where these overlap with each other.

To obtain more precise quantitative information on the features of the object's decomposition parts, we do the following two processes: i) construction of the approximated skeleton $S^{*}$ starting from the available information, i.e., the ordered sequence of spatial coordinates and radii of the vertices, and ii) recovery of the object starting from $S^{*}$. To build a given segment of $S^{*}$, the absolute values of the differences between homologous Cartesian coordinates of the two corresponding extremes of the segment are computed. These are used for: 1) computing the number of unit moves necessary to link the two extremes by means of a digital straight line segment, 2) establishing how many of these moves are towards face-, edge- and vertex- neighbors and 3) establishing the proper way to alternate different moves. To linearly distribute distance values among the voxels in the obtained digital segment, we use the number of voxels in the segment and the absolute value of the difference between the radii of the two extremes. As an example, see Fig. 5 left, showing the approximated skeleton of the skeleton in Fig. 1 middle. The value $\theta=8$ has been used for polygonal approximation. Different colors are used to denote different segments. Centroids and vertices found during polygonal approximation are shown in black.
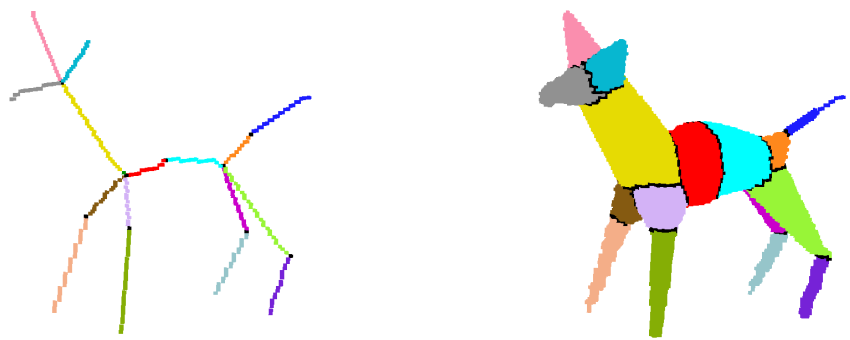

Fig. 5. Approximated skeleton $(\theta=8)$, left, and corresponding object decomposition, right

Once $S^{*}$ has been built, recovery of the simple regions having as spines the segments of $S^{*}$ can be accomplished by applying the reverse distance transformation to the spines. Actually, any two simple regions whose corresponding spines share a common vertex, called hinge, partially overlap. The overlapping region is the ball centered on the hinge. Also simple regions associated to three or more spines sharing the same centroid partially overlap. In this case, the overlapping region is the ball centered on the centroid.

To obtain a decomposition of the object into disjoint components, the overlapping regions (balls centered on the hinges and the centroids) have to be suitably divided among the simple regions sharing them. To reach this goal, we start by applying the reverse distance transformation to $S^{*}$. The obtained distance labeled recovered object is used to order the voxels recovered by $S^{*}$ in decreasing distance order (i.e., from 
those closer to $S^{*}$ to those farther from it), so as to decide on their assignment to the proper object's decomposition component. To this aim, each segment of $S^{*}$ is assigned a different identity label, while all centroids and hinges are assigned a unique common special label. The identity labels of the neighbors of the current recovered object voxel $p$ are checked. If all neighbors of $p$ have the same identity label, also $p$ is assigned that label. Otherwise, $p$ is assigned the special label. Once all recovered voxels have been analyzed, object's components result to labeled with the identity label of their corresponding spines, while surfaces separating adjacent object's components result to be labeled with the special label. Obviously, voxels in the separating surfaces can be assigned to any of the adjacent components. See Fig. 5 right, where voxels with the special label are shown in black.

Depending on whether a meaningful skeleton branch is internal or peripheral, the corresponding region will be a core or a limb. Limbs and cores are elongated regions along which bendings and thickness variations are possible and result to be divided into simple regions.

Differently approximated skeletons can be obtained by using different values for the threshold $\theta$. If the threshold increases, the approximated skeleton represents a rougher version of the object. As an example compare Fig. 5 and Fig. 6. In Fig. 6, the approximated skeleton has been computed by setting $\theta=12$ during polygonal approximation. We may observe that a different number of decomposition components is obtained and that the object in Fig. 6 right is a more schematic representation of the input object.
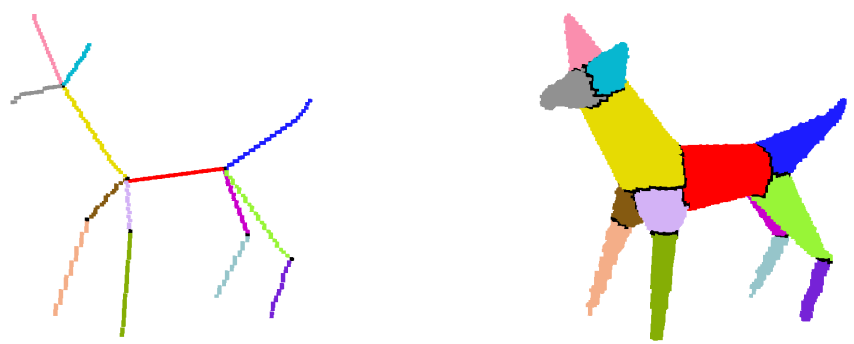

Fig. 6. Approximated skeleton $(\theta=12)$, left, and corresponding object decomposition, right

The decomposition method has been implemented on an Intel Core i7 (3.5 GHz, 8 GB RAM) personal computer and tested on a large set of images taken from publicly available shape repositories, e.g., [6], obtaining in general satisfactory results. The method is computationally advantageous, especially if the approximate evaluation of the features of the simple regions is regarded as sufficient for the specific task. In fact, in this case the part of the process dealing with the construction of the digital segments constituting the approximated skeleton and with the recovery process based on reverse distance transformation and identity label assignment is not necessary.

Two more examples to illustrate the performance of the decomposition method are given in Fig. 7, showing the input objects, the skeletons, the approximated skeletons with $\theta=8$, and the corresponding object decompositions. 

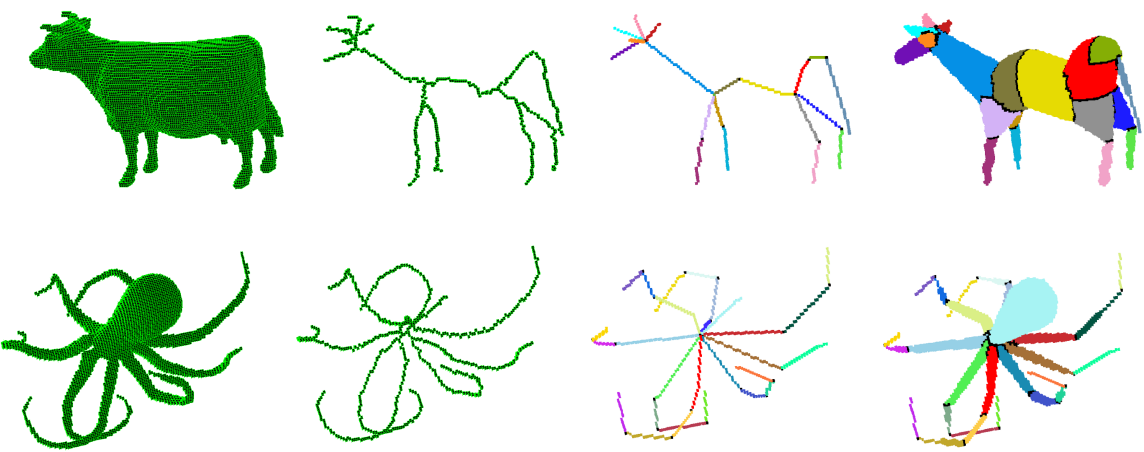

Fig. 7. Each line shows from left to right the input object, the skeleton, the approximated skeleton, and the resulting object decomposition.

\section{Conclusion}

In this paper, decomposition of the curve skeleton of a $3 \mathrm{D}$ object has been used to decompose the object itself. The skeleton is first divided into its constituting branches. Meaningful skeleton branches are detected by using the zones of influence of the branch points. Polygonal approximation in 4D is done so as to divide meaningful skeleton branches into rectilinear segments along which radii change in a linear manner. The regions whose spines are the so obtained segments of the skeleton are sketched versions of the regions into which the object can be interpreted as decomposed. Quantitative information on shape, size, position and orientation of the regions is readily available.

\section{References}

1. Serino, L., Arcelli, C., Sanniti di Baja, G.: 4D polygonal approximation of the skeleton for 3D object decomposition. In: De Marsico, M., Fred, A. (eds.) ICPRAM 2013, pp. 467-472. SCITEPRESS, Lisboa (2013)

2. Arcelli, C., Sanniti di Baja, G., Serino, L.: Distance driven skeletonization in voxel images. IEEE Trans. PAMI 33, 709-720 (2011)

3. Borgefors, G.: On digital distance transform in three dimensions. CVIU 64, 368-376 (1996)

4. Nystrom, I., Borgefors, G.: Synthesising objects and scenes using the reverse distance transformation in 2D and 3D. In: Braccini, C., Vernazza, G., DeFloriani, L. (eds.) ICIAP 1995. LNCS, vol. 974, pp. 441-446. Springer, Heidelberg (1995)

5. Ramer, U.: An iterative procedure for the polygonal approximation of plane curves. CGIP 1, 244-256 (1972)

6. Shilane, P., Min, P., Kazhdan, M., Funkhouser, T.: The Princeton Shape Benchmark. In: Proc. Shape Modeling International SMI 2004, Genova, Italy, pp. 1-12 (2004) 